\title{
Acute Kidney Injury in Lymphoma: A Single Centre Experience
}

\author{
Muhammad Abdul Mabood Khalil, ${ }^{1}$ Hira Latif, ${ }^{1}$ \\ Abdur Rehman, ${ }^{1}$ Waqar Uddin Kashif, ${ }^{1}$ Safia Awan, ${ }^{1}$ Zarghona Khalil, ${ }^{1}$ \\ Uziar Mushtaq, ${ }^{1}$ Maria Ahmad, ${ }^{1}$ Muhammad Ashhad Ullah Khalil, ${ }^{2}$ \\ Manickam Ranga Sami, ${ }^{3}$ and Jackson Tan ${ }^{4}$ \\ ${ }^{1}$ Section of Nephrology, Department of Medicine, Aga Khan University Hospital, Karachi 74800, Pakistan \\ ${ }^{2}$ Department of Medicine, Khyber Teaching Hospital, Peshawar 25000, Pakistan \\ ${ }^{3}$ Department of Nephrology, SSB Hospital, Kuala Belait KA1131, Brunei Darussalam \\ ${ }^{4}$ Department of Nephrology, RIPAS Hospital, Bandar Seri Begawan BA1710, Brunei Darussalam
}

Correspondence should be addressed to Muhammad Abdul Mabood Khalil; doctorkhalil1975@hotmail.com

Received 16 October 2013; Revised 20 December 2013; Accepted 25 December 2013; Published 3 February 2014

Academic Editor: Kazunari Kaneko

Copyright ( 2014 Muhammad Abdul Mabood Khalil et al. This is an open access article distributed under the Creative Commons Attribution License, which permits unrestricted use, distribution, and reproduction in any medium, provided the original work is properly cited.

\begin{abstract}
Background. Acute kidney injury (AKI) is a common but least studied complication of lymphoma. Objective. To determine the frequency and predictors of AKI in lymphoma and to study the impact of AKI on hospital stay and mortality. Methods. Retrospective review of medical records of hospitalized lymphoma patients aged $\geq 14$ years between January 2008 and December 2011 was done. Results. Out of 365 patients, AKI was present in 31.8\% (116/365). Multivariate logistic regression analysis showed that independent predictors for AKI included sepsis (odds ratio (OR) 3.76; 95\% CI 1.83-7.72), aminoglycosides (OR 4.75; 95\% CI 1.15-19.52), diuretics (OR 2.96; 95\% CI 1.31-6.69), tumor lysis syndrome (OR 3.85; 95\% CI 1.54-9.59), and R-CVP regimen (OR 4.70; 95\% CI 1.20-18.36). AKI stages 2 and 3 was associated with increased hospital stay (OR 2.01; 95\% CI 1.19-3.40). Conclusion. AKI was significantly associated with sepsis, aminoglycoside, diuretics, presence of tumor lysis syndrome, and use of R-CVP regimen. Presence of AKIN (Acute Kidney Injury Network) stages 2 and 3 AKI had increased hospital stay. AKI was also associated with increased mortality.
\end{abstract}

\section{Introduction}

Acute kidney injury (AKI) is a well-known complication of cancer $[1,2]$. Etiology of AKI in cancer varies from acute tubular necrosis due to medication or sepsis, volume depletion, tumor lysis syndrome, and obstruction or infiltration of the kidneys. Presence of AKI leads to longer hospitalization and increased cost $[3,4]$. It also increases mortality in patients with cancer $[1,2]$, delays treatment, and increases toxicity of chemotherapy.

Like all cancers, AKI can also occur in lymphoma. The literature reports on AKI in lymphoma are limited to case reports. Lymphoma patients are prone to develop AKI due to acute tubular necrosis secondary to sepsis, nephrotoxic medications, and contrast studies. Lymphomatous infiltration of kidneys [5, 6], light chain proximal tubulopathy [7], membranous nephritis [8], membranoproliferative glomerulonephritis [9], Fanconi syndrome [10], and tumor lysis syndrome [11-13] have all been reported in the literature. This is the first study done on AKI in lymphoma patients and aims to determine frequency and predictors of AKI in lymphoma and to study its impact on hospital stay and mortality.

\section{Materials and Methods}

2.1. Setting and Study Population. This is a retrospective case series study conducted at the Aga Khan University Hospital Karachi, Pakistan. Aga Khan University Hospital is a tertiary care health facility located in the largest city of Pakistan, Karachi, which has a population of around 15 million. It has 15 inpatient units with a total capacity of 563 beds. Data were collected upon approval from the ethics review committee of 
the institution. Study subjects included inpatients admitted with a primary diagnosis of lymphoma. They were identified from a central computerized record for a period of 4 years from January 2008 to December 2011 with the help of the Department of Health Information Management System. Records of the cases were retrieved through codes using International Classification of Diseases 9th Revision Clinical Modification (ICD-9 CM) 201.90-201.98 for Hodgkin lymphoma and 202.80-202.88 for non-Hodgkin lymphoma. Study subjects included patients aged $\geq 14$ years admitted with the primary diagnosis of lymphoma, irrespective of type or stage of disease. Patients with chronic kidney disease were excluded. Patients were divided into two cohorts (those with and those without AKI) in order to determine independent predictors of AKI. Similarly, length of stay and mortality inside hospital were also compared in these two cohorts. Data on demographics, clinical features, laboratory data, length of stay, recovery of renal functions, and mortality were noted. We used the Acute Kidney Injury Network (AKIN) definition for classification of AKI stages [14]. AKI stage 1 was defined as an increase in serum creatinine $>26.5 \mu$ mole $/ \mathrm{L}(\geq 0.3 \mathrm{mg} / \mathrm{dL})$ or 1.5 - to 2 -fold increase from baseline. AKI stage 2 was defined as an increase in serum creatinine of $>2$-3-fold from baseline. AKI stage 3 was defined as an increase in serum creatinine of $>3$-fold from baseline or an absolute serum creatinine of $>354 \mu \mathrm{mol} / \mathrm{L}(\geq 4 \mathrm{mg} / \mathrm{dL})$.

2.2. Definitions. Sepsis was defined as suspected or confirmed infection with the presence of two of the following:

(1) temperature $>38^{\circ} \mathrm{C}$ or $<36.5^{\circ} \mathrm{C}$;

(2) heart rate $>90$ per minute;

(3) respiratory rate $>30$ per minute;

(4) leukocyte count $>11000$ or $<4000$.

Tumor lysis syndrome (Cairo-Bishop definition) was defined as 2 of the following:

(1) uric acid $>8 \mathrm{mg} / \mathrm{dL}$ or $25 \%$ increase from the baseline;

(2) potassium $>6 \mathrm{mmol} / \mathrm{L}$ or $25 \%$ increase from the base line;

(3) phosphate $>4.5 \mathrm{mg} / \mathrm{dL}$;

(4) calcium $<7 \mathrm{mg} / \mathrm{dL}$ or $25 \%$ decrease from the baseline 3 days before or 7 days afterward.

2.3. Data Analysis. Descriptive statistics were used to summarize baseline values and demographic data. Quantitative data were expressed as mean, median, interquartile range and standard deviation (SD) and number of observations with percentage (\%), respectively. Evaluation of association between outcomes (AKI and without AKI group, length of hospital stay) and potential causative factors were used with $\chi^{2}$-test or Fisher's exact tests of independence and Student's $t$ test. Odds ratios (OR) and their 95\% confidence intervals (CI) were estimated using binary logistic regression, with AKI and length of hospital stay ( $\leq 7$ versus $>7$ days) as outcomes. Multivariable models were constructed, including variables that
TABLE 1: Demographic characteristics of study population $(n=365)$.

\begin{tabular}{lc}
\hline Age, years, mean \pm SD & $50.3 \pm 16.3$ \\
Gender, number (\%) & $262(71.8)$ \\
$\quad$ Male & $103(28.2)$ \\
Female & \\
Lymphoma, number (\%) & $87(23.8)$ \\
$\quad$ HL (Hodgkin lymphoma) & $278(76.2)$ \\
$\quad$ NHL (non-Hodgkin lymphoma) & $23(6.3)$ \\
Mechanical ventilation, number (\%) & $31(8.5)$ \\
Vasopressors, number (\%) & $40(11)$ \\
Diuretics, number (\%) & $34(9.3)$ \\
Vancomycin, number (\%) & $16(4.4)$ \\
Amphotericin B, number (\%) & $14(3.8)$ \\
Aminoglycosides, number (\%) & $0.8(0.4-11.1)$ \\
Median baseline creatinine & $2(0-45)$ \\
Median (range) length of stay & $12(3.3)$ \\
Renal replacement therapy (\%) & $28(7.7)$ \\
Tumor lysis syndrome (\%) & $64(17.5)$ \\
Sepsis & $116(31.8)$ \\
& AKI stage $1=78(67.2 \%)$ \\
AKI & AKI stage 2=25 (21.6\%) \\
& Aki stage $3=13(11.2 \%)$ \\
Mortality (\%) & $34(9.3 \%)$ \\
\hline
\end{tabular}

showed an effect on the prediction of AKI and length of hospital stay $>7$ days in the univariate analysis. All $P$ values were based on two-sided tests and significance was set at a $P$ value $<0.05$. The analyses were performed using SPSS version 19 .

\section{Results}

3.1. General Characteristics of the Study Population. Out of 365 patients, two-thirds $(71.8 \%)$ were male. Mean age was $50.13 \pm 16.3$ years. One hundred and sixteen $(31.8 \%)$ patients had AKI. Among patients with AKI, 78 (67.2\%) had AKIN stage 1, $25(21.6 \%)$ had AKIN stage 2, and $13(11.2 \%)$ had AKIN stage 3. Sepsis was found in 64 (17.5\%) and tumor lysis in $28(7.7 \%)$ of the patients. Furosemide, vancomycin, amphotericin, and aminoglycoside were used in 40 (11\%), $34(9.31 \%), 16(4.4 \%)$, and $14(3.8 \%)$ patients, respectively. Overall hospital mortality was $9.3 \%$ in AKI patients (Table 1).

3.2. Independent Predictors for AKI. We analyzed various variables including antimicrobial and chemotherapeutic agents used. Various chemotherapy regimen used included R-CHOP (rituximab-cyclophosphamide, hydroxy daunorubicin, oncovin, and prednisolone), CHOP (cyclophosphamide, hydroxy daunorubicin, oncovin, prednisolone), RCVP (rituximab-cyclophosphamide, vincristine, and prednisolone), CVP (cyclophosphamide, vincristine, and prednisolone), ABVD (adriamycin, bleomycin, vinblastine, and dacarbazine) and DHAP (dexamethasone, high dose Ara C, 
TABLE 2: Univariate analysis of factors associated with progression of AKI $(n=365)$.

\begin{tabular}{|c|c|c|c|}
\hline & AKI, $n=116$ & No AKI, $n=249$ & $P$ value \\
\hline Age, in years & $53.7 \pm 15.5$ & $48.7 \pm 16.5$ & 0.005 \\
\hline \multicolumn{4}{|l|}{ Gender } \\
\hline Male & $82(70.7)$ & $180(72.3)$ & 0.75 \\
\hline Female & $34(29.3)$ & $69(27.7)$ & \\
\hline Hospital stay, median (range) & $3(1-17)$ & $3(1-14)$ & $0.62^{*}$ \\
\hline Baseline Cr, median (range) & $0.8(0.4-2.6)$ & $0.8(0.4-11.1)$ & $0.39^{*}$ \\
\hline Maximum Cr, median (range) & $2(0.9-11.3)$ & $1(0.6-25.9)$ & $<0.001^{*}$ \\
\hline \multicolumn{4}{|l|}{ Stage of lymphoma } \\
\hline I & $4(9.3)$ & $9(9.1)$ & 0.17 \\
\hline II & $7(16.3)$ & $32(32.3)$ & \\
\hline III & $10(23.3)$ & $24(24.2)$ & \\
\hline IV & $22(51.2)$ & $34(34.3)$ & \\
\hline Diuretics & $24(20.7)$ & $16(6.4)$ & $<0.001$ \\
\hline Vancomycin & $17(14.7)$ & $17(6.8)$ & 0.02 \\
\hline Amphotericin B & $7(6)$ & $9(3.6)$ & 0.28 \\
\hline Aminoglycosides & $11(9.5)$ & $3(1.2)$ & $<0.001$ \\
\hline Mechanical ventilation & $12(10.3)$ & $11(4.4)$ & 0.03 \\
\hline Vasopressor & $16(13.8)$ & $15(6)$ & 0.01 \\
\hline \multicolumn{4}{|l|}{ Chemotherapy } \\
\hline R-CHOP & $26(22.4)$ & $68(27.3)$ & 0.31 \\
\hline $\mathrm{CHOP}$ & $26(22.4)$ & $60(24.1)$ & 0.79 \\
\hline R-CVP & $13(11.2)$ & $8(3.2)$ & 0.004 \\
\hline CVP & $8(6.9)$ & $11(4.4)$ & 0.32 \\
\hline ABVD & $12(10.3)$ & $42(16.9)$ & 0.11 \\
\hline DHAP & $17(14.7)$ & $16(6)$ & 0.009 \\
\hline Sepsis & $38(57.6)$ & $26(17.2)$ & $<0.001$ \\
\hline Tumor lysis & $18(15.5)$ & $10(4.0)$ & $<0.001$ \\
\hline \multicolumn{4}{|l|}{ Hospital stay } \\
\hline$\leq 7$ days & $72(62.1)$ & $202(81.1)$ & $<0.001$ \\
\hline$>7$ days & $44(37.9)$ & 47 (18.7) & \\
\hline Mortality & $17(14.7)$ & $17(6.8)$ & 0.02 \\
\hline
\end{tabular}

* Mann-Whitney $U$ test.

and cisplatin). R-CHOP, $\mathrm{CHOP}$, and ABVD were the main regimens used in 94,86 , and 52 patients, respectively. Univariate analysis revealed increased age $(P 0.005)$, diuretics $(P<0.001)$, vancomycin $(P 0.02)$, aminoglycoside $(P<$ $0.001)$, R-CVP ( $P$ 0.004), DHAP $(P$ 0.009), sepsis $(P<$ $0.001)$, and tumor lysis $(P<0.001)$ as positive predictors of AKI. However, multivariate logistic regression analysis showed that independent predictors for AKI were sepsis (OR 3.76; 95\% CI 1.83-7.72), aminoglycosides (OR 4.75; 95\% CI $1.15-19.52$ ), tumor lysis syndrome (OR 3.85; 95\% CI 1.549.59), and R-CVP regimen (OR 4.70; 95\% CI 1.20-18.36) (Tables 2 and 3 ).

3.3. Impact on Hospital Stay, Mortality, and Outcome at Discharge. Patients with AKI stages 2 and 3 had statistically increased hospital stay of greater than 7 days when compared to those without AKI (OR 2.01; 95\% CI 1.19-3.40). Mortality was also high in patients with AKI (14.7\%) when compared to those without AKI (6.8\%) (P 0.03) (Table 4). Patients with AKI who died were significantly associated with tumor lysis
TABle 3: Factors predicting AKI.

\begin{tabular}{lcc}
\hline & OR $[95 \%$ CI $]$ & $P$ value \\
\hline Diuretic & & \\
$\quad$ No & 1.0 & \\
Yes & $2.96[1.31-6.69]$ & 0.009 \\
Aminoglycoside & & \\
$\quad$ No & 1.0 & 0.03 \\
Yes & $4.75[1.15-19.52]$ & \\
Sepsis & 1.0 & \\
No & $3.76[1.83-7.72]$ & $<0.001$ \\
Yes & 1.0 & \\
Tumor lysis syndrome & & \\
No & $3.85[1.54-9.59]$ & 0.004 \\
Yes & & 0.02 \\
R-CVP & 1.0 & \\
No & $4.70[1.20-18.36]$ & \\
Yes & & \\
\hline
\end{tabular}


TABLE 4: Impact of AKI on hospital stay and mortality.

\begin{tabular}{lccc}
\hline AKI stages 2, $3>7$ days & No AKI $>7$ days & $\begin{array}{c}\text { Length of hospital stay } \\
\text { Univariate analysis }\end{array}$ & Multivariate analysis \\
\hline $21(48.83 \%)$ & $49(19.52 \%)$ & $P<0.001$ & Mor $2.01(1.19,3.40)], P 0.008$ \\
\hline & No AKI & Mortality & Mnivariate analysis \\
AKI & $17(6.8)$ & $P 0.02$ & \\
\hline $17(14.7)$ &
\end{tabular}

TABLE 5: Factors associated with mortality in AKI.

\begin{tabular}{lccc}
\hline Outcome & Death $(n=17)$ & Alive $(n=99)$ & $P$ value \\
\hline Age, in years & $54.3 \pm 16$ & $53.6 \pm 15.4$ & \\
Gender & & $74(74.7)$ \\
$\quad$ Male (\%) & $8(47.1)$ & $25(25.3)$ & 0.85 \\
Female (\%) & $9(52.9)$ & $10(10.1)$ & 0.04 \\
Tumor lysis (\%) & $8(47.1)$ & $4(4)$ & 0.001 \\
Ventilation (\%) & $8(47.1)$ & $7(15.2)$ & $7.1)$ \\
Diuretic (\%) & $9(52.9)$ & $21(42.9)$ \\
Vasopressor (\%) & $9(52.9)$ & $0.84 \pm 0.29$ \\
Sepsis (\%) & $17(100)$ & & $<.001$ \\
Baseline creatinine & $0.84 \pm 0.22$ & $3(8.1)$ \\
Stage of disease & & $6(16.2)$ \\
I (\%) & $1(16.7)$ & $10(27)$ \\
II (\%) & $1(16.7)$ & $18(48.6)$ \\
III (\%) & 0 & 0.99 \\
IV (\%) & $4(66.7)$ & 0.001 \\
\hline
\end{tabular}

TABLE 6: Outcome of AKI at discharge.

\begin{tabular}{lc}
\hline Death & $17(14.7 \%)$ \\
Full recovery & $45(38.79 \%)$ \\
Partial recovery with no need of dialysis & $40(34.48 \%)$ \\
Persisting AKI with need of dialysis & $14(12.06 \%)$ \\
\hline
\end{tabular}

( $P$ 0.001), need of mechanical ventilation $(P<0.001)$, diuretics $(P$ 0.001), and sepsis $(<0.001)$ (Table 5$)$.

Mortality in our cohort was $14.7 \%$. Forty-three patients (37.06\%) with AKI fully recovered. Around 40 patients (34.48\%) had persisting AKI at discharge but did not need dialysis. However 14 (12.06\%) patients who needed dialysis were still requiring dialysis at the time of discharge (Table 6).

\section{Discussion}

AKI is a well-known complication of cancer. However, very little is known about AKI in lymphoma. To the best of our knowledge, this is the largest report of AKI in lymphoma patients. Our study revealed the presence of AKI in 31.8\% of patients. The incidence of AKI in various cancers has been reported to be between 12 and 49\% [15-19]. Our study incidence is higher than that of Christiansen et al., who reported incidence of AKI (defined as 50\% elevation of baseline serum creatinine) in $29.39 \%$ lymphoma patients [20]. The discrepancy could be explained by the noninclusion of milder AKI patients in their study.

Our multivariate analysis identified sepsis, aminoglycoside usage, diuretics usage, tumor lysis syndrome, and RCVP usage as potential negative predictors. Cancer patients may be immunocompromised due to multiple factors such as chemotherapy, radiotherapy, impairment of normal leukocyte function, or use of corticosteroids $[21,22]$. The dysregulation of their immune system predisposes patients to sepsis which can lead to unregulated cytokine release and haemodynamic disturbances. AKI usually ensues as a result of alterations in renal perfusion resulting from pro-inflammatory insults.

Diuretics usage may have a direct role in causing kidney injury by exacerbating underlying hypovolemic and haemodynamic state. It can also be argued that patients requiring diuretic therapy are likely to have compromised heart or renal function at baseline and, therefore, more likely to develop and sustain kidney injury. On the other hand, it is possible that diuretic therapy was initiated to maintain or increase urine output in patients who may already have AKI. Aminoglycoside has been implicated in the development of kidney injury in haematological malignancies. This could be related to its direct nephrotoxic effects or through synergistic nephrotoxic 
activities with chemotherapy. The presence of sepsis which may have necessitated the usage of aminoglycoside may also have contributed to the risk of AKI.

We found tumor lysis syndrome in $7.7 \%$ of our study population. Various studies have reported spontaneous tumor lysis syndrome in leukemia and lymphoma [11, 23-26]. Hande kjellstrand and his colleagues found tumor lysis in $42 \%$ of the patients with lymphoma, which is higher than our result [23]. However, unlike our study Hande Kjellstrand et al. included only high grade lymphoma which has a greater propensity for the development of tumor lysis syndrome.

Various chemotherapy regimens were used in our cohort of patients. From our experience, the use of R-CVP regimen was significantly associated with AKI. R-CVP regimen is used for stages III and VI follicular lymphoma. High tumor burden in stages III and IV follicular lymphoma with potential of tumor lysis in combination with cyclophosphamide nephrotoxicity might be a reason for this. Alternatively, combination of cyclophosphamide with vincristine may be more nephrotoxic, which needs further exploration through future studies. AKI in cancers causes significant morbidity and mortality. Lameire et al. reported 3-month mortality of 30 percent [27]. Another study reported six-month mortality of $73 \%$ in critically ill patients with cancer and AKI [28]. Lahoti et al. showed relatively mild elevation of creatinine with worse mortality [29]. We in our study found mortality of $14.7 \%$ in patients with lymphoma complicated with AKI as compared to $6.8 \%$ in those without AKI. Similarly, presence of AKI stages 2 and 3 in patients with lymphoma had more propensity for a prolonged hospital stay.

We speculate that tumor lysis syndrome, concurrent sepsis with superimposed use of aminoglycosides, and diuretics use make lymphoma patients prone to develop AKI. This in turn results in more mortality and morbidity. Therefore, patients with high grade lymphoma should be cautiously watched during their chemotherapy as they can potentially deteriorate by developing tumor lysis and subsequent AKI. Similarly, sepsis should be vigorously looked for and treated appropriately. While managing gram negative sepsis broad spectrum, antibiotics other than aminoglycosides should be chosen if possible. Patients with sepsis and tumor lysis are often volume depleted and need aggressive fluid resuscitation. Therefore, diuretics use should be judiciously used only in the presence of evident fluid overload. We can thus potentially avoid AKI and hence can decrease mortality and morbidity.

We acknowledge several limitations of our study. This is a retrospective study based on data from a single centre. Due to the retrospective nature of this study, etiology of AKI was difficult to determine. Similarly, due to lack of longterm followup of the patients, long-term impact of AKI on lymphoma patients could not be determined. Multicentre prospective studies with long-term followup are needed to analyze etiology of AKI, its long-term outcome, and impact on mortality and morbidity. Similarly, there is a need to determine least nephrotoxic chemotherapy regimen through prospective analysis. Judging by the results of this study, we propose the following management considerations for highrisk lymphoma patients:
(1) careful attention to haemodynamics and volume status;

(2) avoidance of specific drugs;

(3) avoidance of tumour lysis with prophylactic measures (e.g., prophylactic allopurinol/aggressive rehydration, etc.);

(4) early identification and treatment of sepsis;

(5) choice of chemotherapy;

(6) early initiation of RRT (? debatable);

(7) early involvement of nephrology team;

(8) early involvement of intensivists.

\section{Conclusions}

AKI is common in lymphoma. Tumor lysis syndrome, sepsis, and use of aminoglycoside and furosemide were associated with development of AKI. Presence of AKI stages 2 and 3 resulted in increased mortality and prolonged hospital stay. We feel that identification of these negative predictors and early intervention can help to prevent or reduce the severity of AKI.

\section{Abbreviations}

AKI: Acute kidney injury

AKIN: Acute Kidney Injury Network

R-CHOP: Rituximab-cyclophosphamide, hydroxy daunorubicin, oncovin, and prednisolone

CHOP: Cyclophosphamide, hydroxy daunorubicin, oncovin, and prednisolone

R-CVP: Rituximab, cyclophosphamide, vincristine, and prednisolone

CVP: Cyclophosphamide, vincristine, and prednisolone

ABVD: Adriamycin, bleomycin, vinblastine, and dacarbazine

DHAP: Dexamethasone, high dose Ara C, and cisplatin.

\section{Conflict of Interests}

The authors declare that they have no conflict of interests regarding the publication of this paper.

\section{References}

[1] M. Darmon, M. Ciroldi, G. Thiery, B. Schlemmer, and E. Azoulay, "Clinical review: specific aspects of acute renal failure in cancer patients," Critical Care, vol. 10, no. 2, article 211, 2006.

[2] J. M. Evison, P. Rickenbacher, R. Ritz et al., "Intensive care unit admission in patients with haematological disease: incidence, outcome and prognostic factors," Swiss Medical Weekly, vol. 131, no. 47-48, pp. 681-686, 2001.

[3] G. M. Chertow, E. Burdick, M. Honour, J. V. Bonventre, and D. W. Bates, "Acute kidney injury, mortality, length of stay, and costs in hospitalized patients," Journal of the American Society of Nephrology, vol. 16, no. 11, pp. 3365-3370, 2005. 
[4] J. F. Dasta, S. L. Kane-Gill, A. J. Durtschi, D. S. Pathak, and J. A. Kellum, "Costs and outcomes of acute kidney injury (AKI) following cardiac surgery," Nephrology Dialysis Transplantation, vol. 23, no. 6, pp. 1970-1974, 2008.

[5] A. M. Alkhunaizi, R. A. Daabil, and M. F. Dawamneh, "Acute kidney injury secondary to lymphomatous infiltration and the role of kidney biopsy," Saudi Medical Journal, vol. 29, no. 12, pp. 1808-1810, 2008.

[6] S. Al-Salam, A. Shaaban, M. Alketbi, N. U. Haq, and S. Abouchacra, "Acute kidney injury secondary to renal large Bcell lymphoma: role of early renal biopsy," International Urology and Nephrology, vol. 43, no. 1, pp. 237-240, 2011.

[7] S. Rane, S. Rana, M. Sachdeva, and K. Joshi, "Light chain proximal tubulopathy without crystals in a case of Burkitt lymphoma presenting with acute kidney injury," American Journal of Kidney Diseases, vol. 62, no. 3, pp. 638-641, 2013.

[8] M. Kayataş, G. Yıldız, M. Timuçin, F. Candan, E. Yıldız, and M. Sencan, "A case of acute renal failure caused by Hodgkin's lymphoma: concurrent membranous glomerulonephritis and interstitial HL-CD 20 lymphoid infiltration," Renal Failure, vol. 33, no. 3, pp. 363-366, 2011.

[9] A. Lubas, A. Mróz, J. Smoszna, and S. Niemczyk, "Membranoproliferative glomerulonephritis, mantle cell lymphoma infiltration, and acute kidney injury," International Urology and Nephrology, vol. 45, no. 5, pp. 1489-1494, 2013.

[10] W. Ye, B. Han, B. Liu et al., "Non-Hodgkin's lymphoma primarily presenting with fanconi syndrome and acute kidney injury," Chinese Medical Sciences Journal, vol. 25, no. 3, pp. 185-188, 2010.

[11] K. R. Hande and G. C. Garrow, "Acute tumor lysis syndrome in patients with high-grade non-Hodgkin's lymphoma," American Journal of Medicine, vol. 94, no. 2, pp. 133-139, 1993.

[12] S. Alkhuja and H. Ulrich, "Acute renal failure from spontaneous acute tumor lysis syndrome: a case report and review," Renal Failure, vol. 24, no. 2, pp. 227-232, 2002.

[13] J. Barquero Romero, I. Catalina Fernández, C. Hernández Sáez, J. L. Jiménez Morales, and F. García de la Llana, "Spontaneous tumor lysis syndrome in a patient with non-Hodgkin's lymphoma," Anales de Medicina Interna, vol. 22, no. 8, pp. 387-389, 2005.

[14] R. L. Mehta, J. A. Kellum, S. V. Shah et al., "Acute Kidney Injury Network: report of an initiative to improve outcomes in acute kidney injury," Critical Care, vol. 11, no. 2, article R31, 2007.

[15] J.-J. Lanore, F. Brunet, F. Pochard et al., "Hemodialysis for acute renal failure in patients with hematologic malignancies," Critical Care Medicine, vol. 19, no. 3, pp. 346-351, 1991.

[16] D. D. Benoit, E. A. Hoste, P. O. Depuydt et al., "Outcome in critically ill medical patients treated with renal replacement therapy for acute renal failure: comparison between patients with and those without haematological malignancies," Nephrology Dialysis Transplantation, vol. 20, no. 3, pp. 552-558, 2005.

[17] E. Azoulay, C. Recher, C. Alberti et al., "Changing use of intensive care for hematological patients: the example of multiple myeloma," Intensive Care Medicine, vol. 25, no. 12, pp. 1395-1401, 1999.

[18] E. Azoulay, D. Moreau, C. Alberti et al., "Predictors of shortterm mortality in critically ill patients with solid malignancies," Intensive Care Medicine, vol. 26, no. 12, pp. 1817-1823, 2000.

[19] M. Darmon, G. Thiery, M. Ciroldi et al., "Intensive care in patients with newly diagnosed malignancies and a need for cancer chemotherapy," Critical Care Medicine, vol. 33, no. 11, pp. 2488-2493, 2005.
[20] C. F. Christiansen, M. B. Johansen, W. J. Langeberg, J. P. Fryzek, and H. T. Sørensen, "Incidence of acute kidney injury in cancer patients: a Danish population-based cohort study," European Journal of Internal Medicine, vol. 22, no. 4, pp. 399-406, 2011.

[21] C. Conlon, "Sepsis in immunocompromised hosts," Journal of the Royal College of Physicians of London, vol. 34, no. 6, pp. 533536, 2000.

[22] G. P. Bodey, "Infection in cancer patients. A continuing association," American Journal of Medicine, vol. 81, no. 1 A, pp. 11-26, 1986.

[23] C. M. Kjellstrand, D. C. Campbell, B. von Hartitzsch, and T. J. Buselmeier, "Hyperuricemic acute renal failure," Archives of Internal Medicine, vol. 133, no. 3, pp. 349-359, 1974.

[24] G. C. Tsokos, J. E. Balow, R. J. Spiegel, and L. T. Magrath, "Renal and metabolic complications of undifferentiated and lymphoblastic lymphomas," Medicine, vol. 60, no. 3, pp. 218229, 1981

[25] J. Veenstra, R. T. Krediet, R. Somers, and L. Arisz, “Tumour lysis syndrome and acute renal failure in Burkitt's lymphoma. Description of 2 cases and a review of the literature on prevention and management," Netherlands Journal of Medicine, vol. 45, no. 5, pp. 211-216, 1994.

[26] A. M. Jasek and H. J. Day, "Acute spontaneous tumor lysis syndrome," American Journal of Hematology, vol. 47, no. 2, pp. 129-131, 1994.

[27] N. H. Lameire, C. D. Flombaum, D. Moreau, and C. Ronco, "Acute renal failure in cancer patients," Annals of Medicine, vol. 37, no. 1, pp. 13-25, 2005.

[28] M. Soares, J. I. F. Salluh, M. S. Carvalho, M. Darmon, J. R. Rocco, and N. Spector, "Prognosis of critically ill patients with cancer and acute renal dysfunction," Journal of Clinical Oncology, vol. 24, no. 24, pp. 4003-4010, 2006.

[29] A. Lahoti, H. Kantarjian, A. K. Salahudeen et al., "Predictors and outcome of acute kidney injury in patients with acute myelogenous leukemia or high-risk myelodysplastic syndrome," Cancer, vol. 116, no. 17, pp. 4063-4068, 2010. 


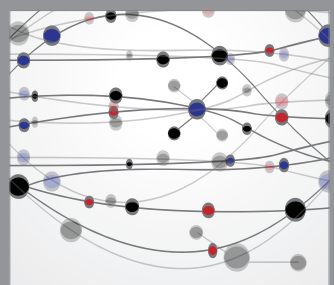

The Scientific World Journal
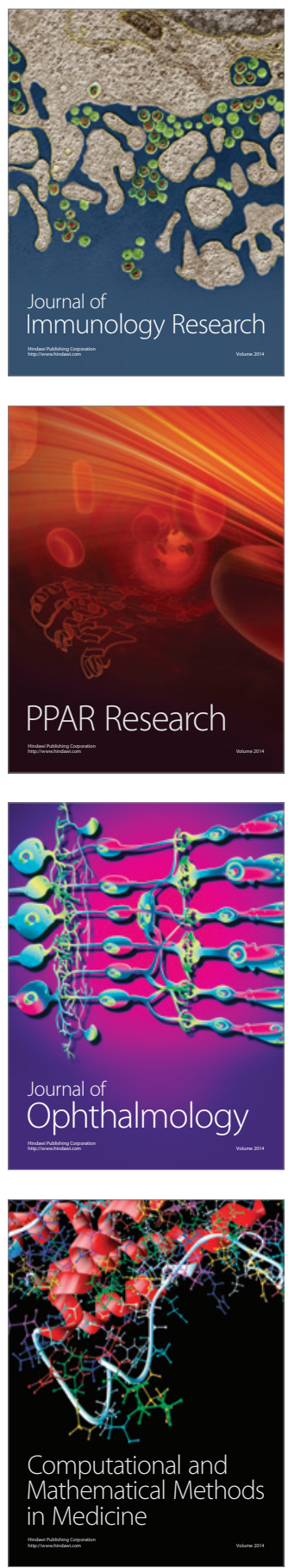

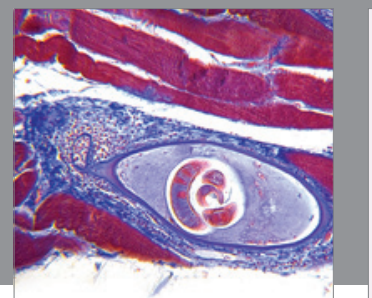

Gastroenterology

Research and Practice
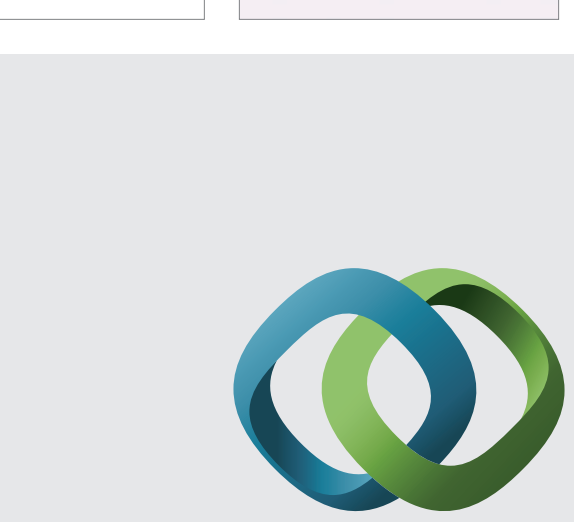

\section{Hindawi}

Submit your manuscripts at

http://www.hindawi.com
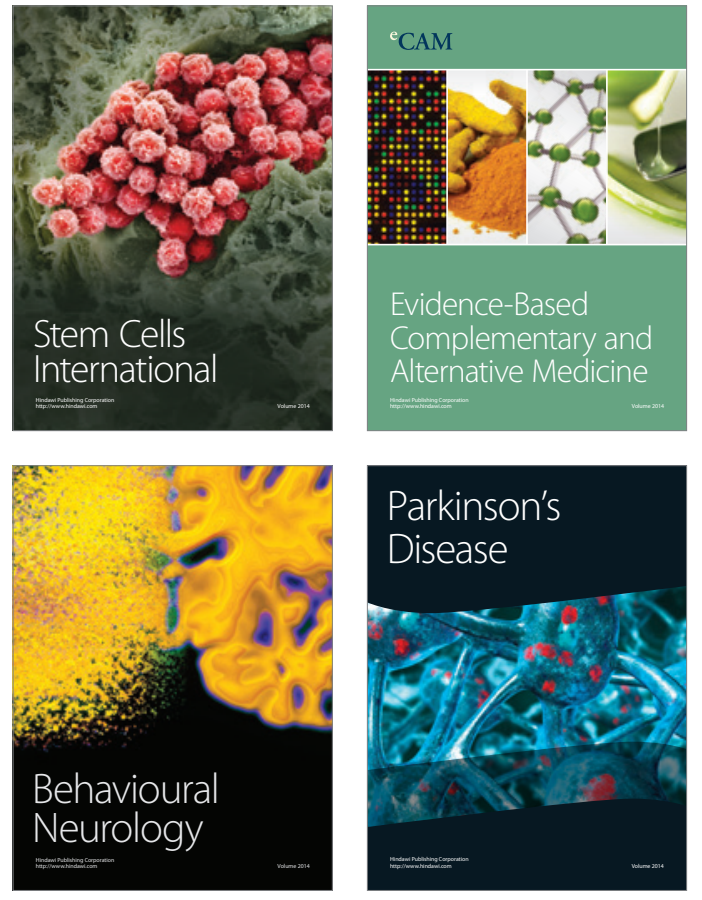
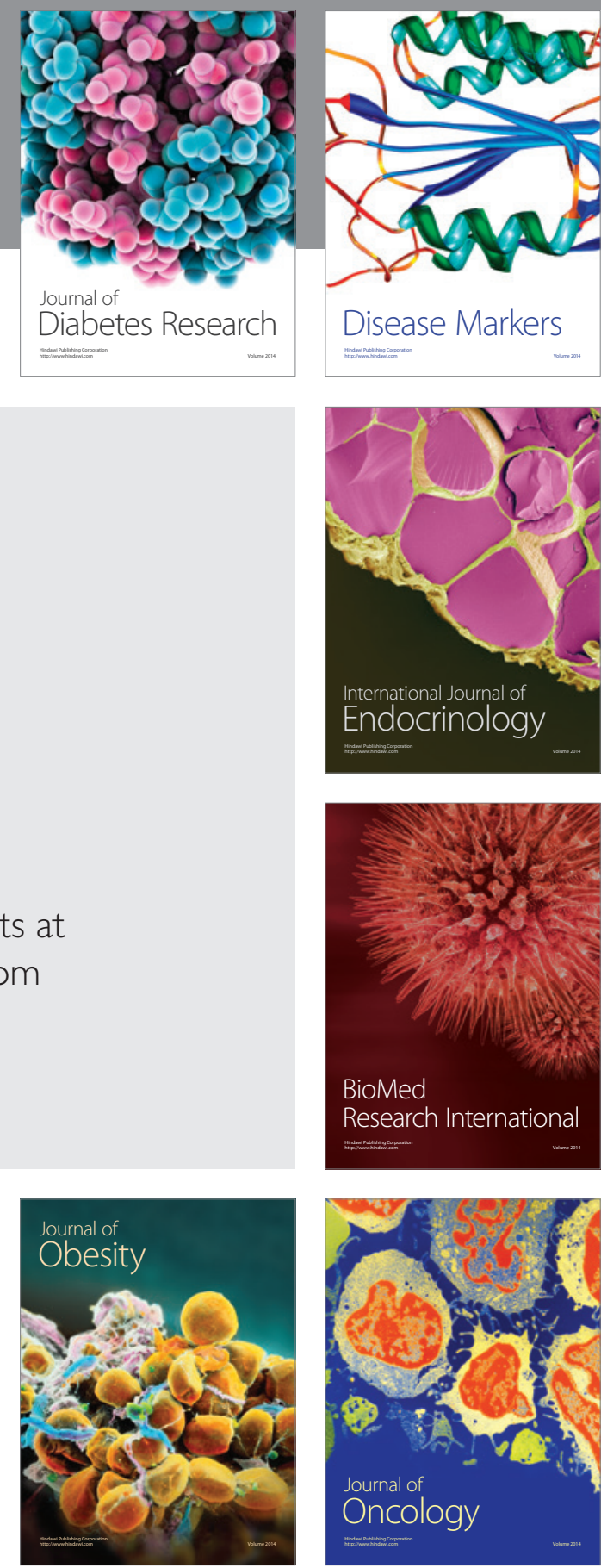

Disease Markers
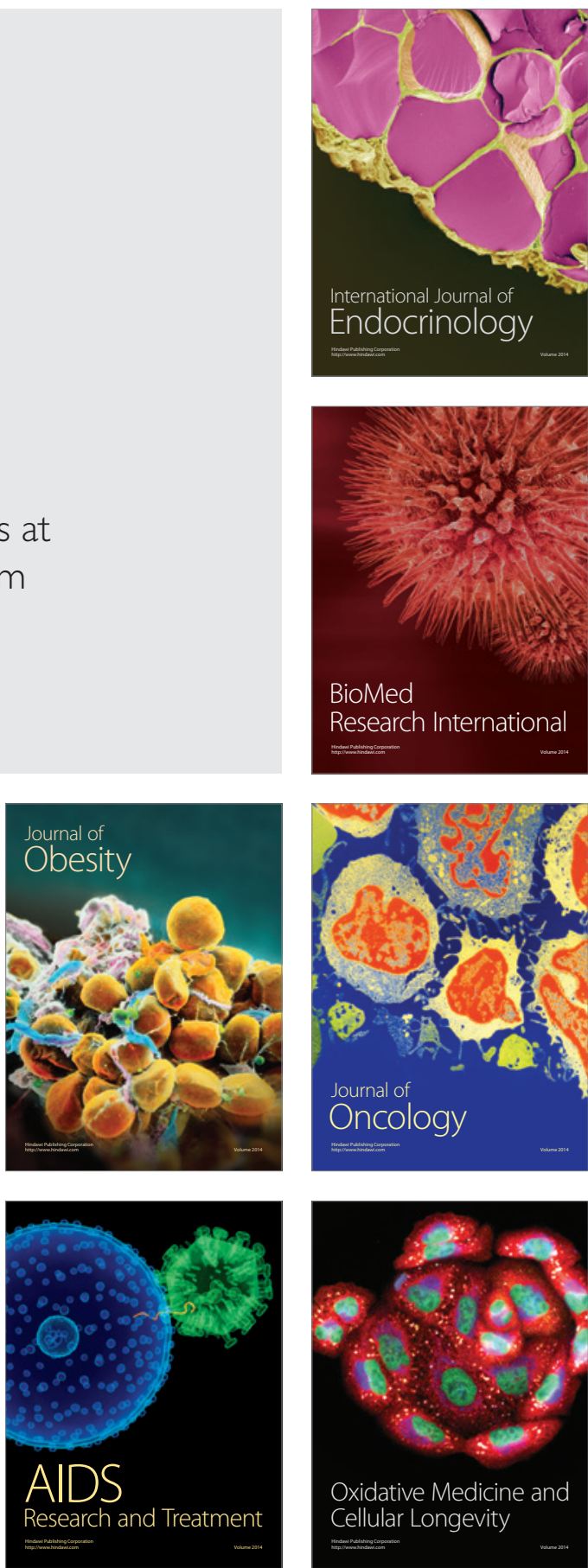